\title{
Spectrum of dystrophin gene mutations observed in patients suspected of Duchenne muscular dystrophy in Pakistan
}

\author{
Author: Zeeshan Ahmed
}

\section{Introduction}

Duchenne muscular dystrophy (DMD) is an inherited X-linked recessive genetic disorder caused by a defect in the dystrophin gene. This results in a quantitative and/or qualitative abnormality in the dystrophin protein causing progressive muscular damage and weakness.

\section{Objective}

To investigate the spectrum of deletions and duplications in the dystrophin gene of patients suspected of DMD or of being DMD carriers.

\section{Methods}

A retrospective review of DMD testing performed from March 2017 to June 2018 was conducted at Aga Khan University Hospital, Karachi. Multiplex ligation-dependent probe amplification (MLPA) was used for identification of deletion/duplication in 79 exons of the dystrophin gene.

\section{Results}

In a total of 92 cases, 85 males and 7 females were examined. In males, deletions and duplications were identified in $44(52 \%)$ of the 85 cases; deletions were found in 38 cases $(45 \%)$ and duplications in 6 cases ( $7 \%$ ). In seven cases referred for females, two of the seven females (30\%) were found to have a heterozygous deletion, suggestive of carrier status.

\section{Conclusions}

This study identifies deletions in dystrophin exon 42-52 to be the most common while duplications were mostly found in exons 3-7. This information can facilitate the use of new treatments with exon-skipping drugs which are mutation specific for DMD.

\section{Conflict of interest statement \\ None declared.}

